\title{
Association of Adiposity, Cardiorespiratory Fitness and Exercise Practice with the Prevalence of Type 2 Diabetes in Brazilian Elderly Women
}

\author{
Maressa P. Krause ${ }^{1}$, Tatiane Hallage ${ }^{2}$, Mirnaluci Paulino Ribeiro Gama ${ }^{3}$, Fredric L. Goss ${ }^{1}$, Robert \\ Robertson ${ }^{1}$, Sergio G. da Silva ${ }^{2}$
}

1. Center for Exercise and Health-Fitness Research - University of Pittsburgh, USA;

2. Sport and Exercise Research Center - Universidade Federal do Paraná, Brazil;

3. Division of Endocrinology and Diabetes - Departamento de Clínica Médica - Hospital Universitário Evangélico de Curitiba, Brazil.

Correspondence to: Maressa Priscila Krause,mpk19@pitt.edu; maressakrause@hotmail.com

Received: 2007.10.02; Accepted: 2007.11.12; Published: 2007.11.21

Background: Diabetes incidence in people with advanced age is increasing at an alarming rate, and for this reason the screening of high-risk individuals such as elderly women is critically important. Objective: To analyze the association of adiposity, cardiorespiratory fitness and exercise practice with type 2 diabetes (T2D) in elderly Brazilian women. Methods: Participated of this cross sectional study 1,059 elderly women (mean 69.5 yr; SD 6.1), who self-reported family history of cardiovascular disease, smoking status, hypertension, and T2D diagnosed previously by a physician. The following independent variables were assessed: exercise practice, body mass index, waist circumference, and cardiorespiratory fitness. Logistic regression analysis was used to investigate the association between each independent variable with T2D using adjusted-models. Results: T2D prevalence was $16 \%$. General and central adiposity were directly associated with T2D, whereas cardiorespiratory fitness was inversely related with T2D. The joint effect of exercise practice and central adiposity showed that inactive women had higher odds ratio for T2D when compared with active ones, within the same WC group. Inactive women with WC $\geq 94.0 \mathrm{~cm}$ had an odds ratio of 5.8 (95\% IC 1.3-25.3). Conclusions: A direct positive association was found between general and central adiposity, as well as an inverse relation between CRF and exercise practice with T2D. Elderly women who practice exercise regularly had lower odds for T2D. Health professionals should encourage individuals of all ages to engage on regular exercise practice, which could reduce body fatness and may be beneficial in reducing the prevalence of T2D in older ages.

Key words: Adiposity; cardiorespiratory fitness; exercise and type 2 diabetes.

\section{Introduction}

Type 2 diabetes (T2D) and its related obesity comorbidities are a significant and growing public health problem $[1,2,3]$. Factors responsible for the increased prevalence of T2D have been the target of many studies. Family history, excess of body fat, and physical inactivity have been linked to T2D [4].

Excess of adiposity, specifically in the central region (i.e. visceral adiposity), is strongly associated with the prevalence of $\mathrm{T} 2 \mathrm{D}$, the increase of the peripheral insulin resistance, and the decrease of glucose sensitivity [5-10].

Lifestyle characteristics, such as leisure time physical activity has been inversely related to T2D and metabolic syndrome [3, 11, 12]. A similar inverse association with the risk for T2D has been documented regarding cardiorespiratory fitness $(\mathrm{CRF})$, which is developed and maintained by regular exercise practice independently of age [12-16].

Increasing age is associated with a greater prevalence of impaired glucose tolerance and T2D [17, 18]. There is an interaction of many factors associated with aging which may contribute to the impaired glucose tolerance observed in elderly individuals. These factors include: increased general and central adiposity, decreased physical activity, medications, comorbidities, and insulin secretory dysfunctions [19, 20, 21].

Although many studies have examined the association between adiposity, physical activity or CRF with T2D, only a few studies have specifically targeted elderly women. Furthermore, the influence of exercise practice on the prevalence of T2D in elderly individuals is still unclear [11, 14, 15, 22]. Therefore, the objective of this study was to analyze the association of general and central adiposity, cardiorespiratory fitness and exercise practice with T2D in elderly Brazilian women.

\section{Methods}

\section{Design}

The present study was conducted in the city of Curitiba - Paraná, Brazil. The subjects of this study were elderly women that were participating in community groups, randomly selected, in the entire city. Subjects were invited to participate in this 
investigation from the groups selected, and after receiving a detailed clarification of the procedures involved in this research, including benefits and possible risks, subjects signed the informed consent, indicating their participation as voluntary.

The sample was composed of 1,059, non-institutionalized, women, aged between 60.0-88.8 years (mean 69.5; SD 6.1). Subjects were predominantly white, and were classified as low or middle socio-economic level, $50.0 \%$ reported presence of hypertension, $44.0 \%$ reported family history of cardiovascular disease, and $4.8 \%$ were current smokers.

With the objective to avoid the influence of circadian variations, all the assessments were conducted, between 08:00 and 10:00 am. Furthermore, the participants were instructed not to ingest any food two hours before the tests, as well as to avoid any vigorous physical activity for 24 hours preceding them. All assessments were conducted at the Physiology Laboratory of the Exercise and Sport Research Center of the Universidade Federal do Paraná.

The study protocol was approved by the Ethics Committee of the Universidade Federal do Paraná, according to the norms established in the Resolution 196/96 of the National Health Council concerning research involving human subjects.

\section{Measurements}

In order to avoid inter-examinator variability, all anthropometrics measures were obtained by a single trained examiner. Body mass, height and waist circumference were assessed. Body Mass Index was calculated for each subject.

A 6-min walk test was administered to assess cardiorespiratory fitness [20]. The test was performed on a $54.4 \mathrm{~m}$ rectangular course $(18.0 \mathrm{~m}$ length $\times 9.2 \mathrm{~m}$ width). The maximum distance walked in 6 minutes was recorded for each subject [23].

The exercise practice was determined by the Modified Baecke Questionnaire for Older Adults proposed by Voorrips et al [24]. This questionnaire is composed of three sections: household activities (domestic physical activity - DPA), sports activities (exercise physical activity - EPA) and leisure time activities (leisure physical activity - LPA). The EPA score was used to classify subjects as "active" or "inactive". All examiners were trained in administering the questionnaire to control for inter-examiner variability.

Socioeconomic level was determined by a validated national socioeconomic questionnaire. Participants reported family history of cardiovascular disease (yes or no) and smoking status (current smoker or not). Hypertension was categorized as blood pressure measured by a physician, where systolic blood pressure exceeded $140 \mathrm{mmHg}$, and diastolic blood pressure exceeded $90 \mathrm{mmHg}$. Participants also reported the presence of T2D (yes or no) previously diagnosed by a physician.

\section{Statistical analyses}

The Kolmogorov Smirnov test of normality was used to determine that the distribution of the sample data was parametric. Logistic regression analysis was used to determine the association of general and central adiposity (BMI and WC), cardiorespiratory fitness (CRF), and EPA classification with T2D. T2D was treated as a dichotomous variable (yes/no). BMI cutoff points were used in the univariate analysis, and WC and CRF were divided into quartiles. Odds Ratio (OR) and their $95 \%$ confidence intervals $(95 \% \mathrm{CI})$ were calculated using age (treated as a continuous variable) and adjusted-models, which included the potential confounders' variables - socioeconomic status (treated as a continuous variable), hypertension, family history of cardiovascular disease, and smoking status (all treated as a dichotomous variable). The subsequent models were created with the purpose to analyze the isolated association of each independent variable of this study with T2D. To investigate the combined effect of exercise practice and central adiposity with $\mathrm{T} 2 \mathrm{D}$, the following joint $\mathrm{WC}$ and active/inactive variable were created based on both quartiles of WC and EPA classification: $W C \leq 80 \mathrm{~cm}$ Active and $W C \leq$ $80 \mathrm{~cm}$ Inactive, WC $80.1-86.9 \mathrm{~cm}$ Active and WC 80.1 $86.9 \mathrm{~cm}$ Inactive, WC $87-93.9 \mathrm{~cm}$ Active and WC 87 $93.9 \mathrm{~cm}$ Inactive, $W C \geq 94 \mathrm{~cm}$ Active and $W C \geq 94 \mathrm{~cm}$ Inactive. The $W C \leq 80 \mathrm{~cm}$ Active group was the reference. The significance level was established a priori at $\mathrm{p}<0.05$ for all analysis.

All analyses were performed using Statistical Package for the Social Sciences (SPSS, version 13.0) for Windows.

\section{Results}

The prevalence of T2D in the sample was $16 \%$. Tables 1 and 2 show the results of the univariate logistic regression analysis, demonstrating the isolated association of each independent variable with T2D. When considering general adiposity, the prevalence of T2D was greater in obese women $(22.3 \%)$. There was a direct association between the odds ratio for T2D and the increase of BMI. Overweight women had an odds ratio of 1.5, and obese women had an odds ratio of 2.28. When analyzing the association of central adiposity with $\mathrm{T} 2 \mathrm{D}$, the results indicated that only $6.6 \%$ of women in the lowest WC quartile reported having $\mathrm{T} 2 \mathrm{D}$, whereas the prevalence of $\mathrm{T} 2 \mathrm{D}$ in the highest WC quartile was almost four-folds higher. Women in the highest quartile of WC had an odds ratio of 3.76 for T2D, after all adjustments.

The inverse association between CRF with T2D is shown in table 2 . Women in the lowest CRF quartile $(>330.8 \mathrm{~m})$ had an odds ratio of 2.09 when compared with those in the highest CRF quartile. Women classified as inactive had a greater odds ratio for T2D when compared with active women, with an OR of 1.5.

The joint effect of exercise practice and central adiposity is shown in Figure 1. Active women had a lower odds ratio for T2D when compared with inactive women. Furthermore, active women that presented 
values between $80-86 \mathrm{~cm}$ for WC had an odds ratio of $0.82(95 \%$ CI $0.11-6.25)$ for T2D. However, for the same WC range $(80-86 \mathrm{~cm})$ inactive women had an odds ratio of 4.1 (95\% CI $0.92-18.11)$. In addition, inactive women that had higher central adiposity values (WC $\geq 94.0 \mathrm{~cm}$ ) had an elevated odds ratio for T2D (OR 5.8; 95\% CI 1.32-25.39).

Table 1. Univariate regression analysis models for type 2 Diabetes according to general and central adiposity groups.

\begin{tabular}{|c|c|c|c|c|}
\hline & $\begin{array}{c}\text { T2D } \\
(\%)\end{array}$ & $\begin{array}{c}\text { Model 1 } \\
\text { OR (95\%CI) }\end{array}$ & $\begin{array}{c}\text { Model 2 } \\
\text { OR }(95 \% \mathrm{CI})\end{array}$ & $\begin{array}{c}\text { Model 3 } \\
\text { OR (95\%CI) }\end{array}$ \\
\hline $\begin{array}{c}\text { BMI } \\
\text { Normal }\end{array}$ & 9.1 & 1.0 & & \\
$\begin{array}{c}\text { Overweight } \\
\text { Obese }\end{array}$ & 14.6 & 1.69 & 1.0 & 1.0 \\
& 22.3 & $(1.02-2.82)$ & $(0.95-2.66)$ & $(0.90-2.55)$ \\
\hline & & $(1.71-4.69)$ & $(1.52-4.28)$ & $(1.35-3.85)$ \\
\hline WC $(\mathrm{cm})$ & & & & \\
$\leq 80$ & 6.6 & 1.0 & 1.0 & 1.0 \\
$80.1-86.9$ & 14.1 & 2.31 & 2.15 & 2.10 \\
$87.0-93.9$ & 19.0 & $(1.29-4.12)$ & $(1.20-3.85)$ & $(1.17-3.78)$ \\
$\geq 94.0$ & 24.7 & 3.29 & 3.05 & 2.89 \\
& & $(1.85-5.86)$ & $(1.70-5.46)$ & $(1.60-5.19)$ \\
& & 4.60 & 4.05 & 3.76 \\
& & $(2.63-8.03)$ & $(2.30-7.14)$ & $(2.12-6.68)$ \\
\hline
\end{tabular}

Model 1 - adjusted for age

Model 2 - adjusted for age and confounders (socioeconomic status, hypertension, family history for CVD and smoking status)

Model 3 - Adjusted for age, confounders, EPA and CRF

Table 2. Univariate regression analysis models for type 2 Diabetes according to cardiorespiratory fitness and exercise practice groups.

\begin{tabular}{|c|c|c|c|c|c|}
\hline & $\begin{array}{l}\text { T2D } \\
(\%)\end{array}$ & $\begin{array}{c}\text { Model } 1 \\
\text { OR } \\
(95 \% \mathrm{CI})\end{array}$ & $\begin{array}{c}\text { Model } 2 \\
\text { OR } \\
(95 \% \mathrm{CI})\end{array}$ & $\begin{array}{c}\text { Model } 3 \\
\text { OR } \\
(95 \% \mathrm{CI})\end{array}$ & $\begin{array}{c}\text { Model } 4 \\
\text { OR } \\
(95 \% \mathrm{CI})\end{array}$ \\
\hline $\begin{array}{c}\text { CRF }(\mathrm{m}) \\
>490.2 \\
\\
431.1-490.1 \\
330.9-431.0 \\
<330.8\end{array}$ & $\begin{array}{l}10.4 \\
12.0 \\
19.6 \\
21.0\end{array}$ & $\begin{array}{c}1.0 \\
1.18 \\
(0.67-2.07) \\
2.11 \\
(1.26-3.53) \\
2.30 \\
(1.37-3.84)\end{array}$ & $\begin{array}{c}1.0 \\
1.17 \\
(0.66-2.05) \\
1.98 \\
(1.18-3.34) \\
2.19 \\
(1.30-3.58)\end{array}$ & $\begin{array}{c}1.0 \\
1.12 \\
(0.63-1.99) \\
1.85 \\
(1.08-3.15) \\
2.09 \\
(1.21-3.58)\end{array}$ & $\begin{array}{l}- \\
- \\
-\end{array}$ \\
\hline $\begin{array}{l}\text { EPA } \\
\text { Active } \\
\text { Inactive }\end{array}$ & $\begin{array}{l}13.9 \\
16.5\end{array}$ & $\begin{array}{c}1.0 \\
1.18 \\
(0.75-1.85)\end{array}$ & $\begin{array}{c}1.0 \\
1.22 \\
(0.77-1.92)\end{array}$ & - & $\begin{array}{c}1.0 \\
1.56 \\
(0.97-2.52)\end{array}$ \\
\hline
\end{tabular}

Model 1 - adjusted for age

Model 2 - adjusted for age and confounders (socioeconomic status, hypertension, family history for CVD and smoking status)

Model 3 - Adjusted for age, confounders, EPA, BMI and WC. Model 4 - Adjusted by age, confounders, BMI and CRF.

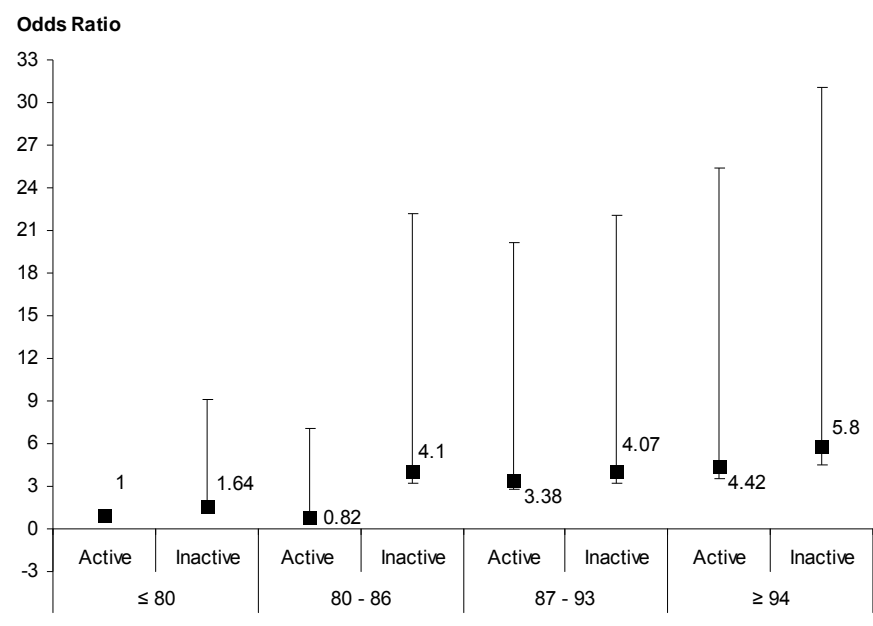

Waist Circumference (cm)

Figure 1. Joint relation of exercise practice and central adiposity with the odds ratio of incident Diabetes. Error bars indicate 95\% confident interval. Adjusted for age, confounders (socioeconomic status, hypertension, family history for CVD and smoking status), socioeconomic status, hypertension, family history for CVD, smoking status, and CRF.

\section{Discussion}

The prevalence of T2D has been the focus of recent research in many countries, however, there are no recent investigations involving the Brazilian population. The last available data in Brazil was published in 1998, showing that the prevalence of diabetes was $17.4 \%$ for elderly (60-69 years), and $7.6 \%$ for males and females subjects with 30-69 years [25]. Therefore, the findings of this study highlight the importance to investigate the factors associated with T2D that can help Brazilian health professionals to amplify their knowledge about this matter, and thus influencing them to develop new strategies involving primary and secondary prevention.

The findings presented here are supported by other investigations which showed that general and central adiposity as well as physical inactivity can increase the risk for T2D. On the other hand, CRF is inversely related to T2D. In addition, this relation has also been noted between CRF with other clinical conditions such as obesity, metabolic syndrome, cardiovascular and coronary heart disease $[3,11,13-15$, 22, 26-28].

A representative American research that focused on a similar approach was the Medical Expenditure Panel Survey (MEPS) that collected socio-demographic and health status data from approximately 68,500 adults from U.S., with the purpose of verifying the prevalence of obesity, inactivity and T2D. The results of the MEPS project showed an increase in the prevalence of T2D with aging, in which $15.9 \%$ males and females subjects aged 70 years or older had T2D. In addition, a positive relation was found between BMI with T2D, whereas an inverse relation was noted with an "active" lifestyle. Active subjects, even when overweight or obese (classes I, II and III), had a lower 
risk for T2D [3]. Weinstein et al [11] also found that general adiposity and physical activity are independent predictors of the prevalence of T2D. The combined analysis using BMI and "active" or "inactive" categories showed greater hazard ratios in inactive subjects for all BMI categories, as well as a progressive increase in the hazard ratio for each BMI category, with higher values for obese and inactive subjects (HR 11.8; 95\% CI 8.75-16.0).

It is well established that the increase of body mass leads to an increase of the risk for T2D and cardiovascular diseases (relative ratio of 1.76; 95\% CI 1.16-2.67, and relative ratio of 1.32; 95\%CI 1.107-1.62; $\mathrm{p}<0.01$, respectively); conversely, it seems that a decrease in body mass could reduce this risk [28]. For this reason, it is expected that people who engaged in regular exercise practice tend to present a lower odds to have T2D, by maintaining their body weight and CRF than those that have an inactive lifestyle [22].

Furthermore, the positive impact of an active lifestyle on the presence of other clinical conditions is widely reported. Franks et al [14] reported a strong inverse relation between both physical activity energy expenditure (PAEE) and CRF with metabolic syndrome, indicating that the maintenance of higher physical activity levels could act as a primary prevention for metabolic diseases, whereas low CRF is associated with the increased risk for cardiovascular diseases mortality. On the other hand, high CRF decreased the risk for metabolic syndrome in approximately $65-75 \%$. High CRF can be considered a protective effect to premature death, independent of general adiposity or the presence of metabolic disturbs $[13,22]$, as well as it could attenuate the risk for metabolic syndrome independent of central adiposity [27].

Additionally, an inverse relation between metabolic disturbs with physical activity level was reported by Laaksonen et al [15]. Unfit individuals, who engaged in vigorous physical activity for less than ten minutes per week, were at a higher risk for metabolic syndrome when compared with fit individuals who engaged in at least 60 minutes per week of vigorous activity.

These previous studies reflect the consensus in the scientific literature about how excess of adiposity adversely affects health status, and how physical activity and CRF are beneficial even for individuals with excess of fatness [3, 22, 27]. Regular exercise practice can result in a positive modification in fitness and body composition, consequently, contributing to a decrease in the risk for T2D, other morbidities and mortality.

Diabetes incidence in people with advanced age is increasing at an alarming rate [1-3], and for this reason the screening of high-risk individuals such as elderly women is critically important because it allows better understanding, monitoring of this condition and comorbidities associated with it. Elderly people who have diabetes could become more vulnerable to other chronic conditions associated with metabolic and cardiovascular dysfunction, such as high levels of triglycerides and C-reactive protein, lower levels of HDL, silent myocardial ischemia, neuropathy, peripheral arterial insufficiency, myocardial infraction, macrovascular disease, abnormal myocardial perfusion among others $[17,29,30]$.

Elderly people who practice exercises regularly have a better health status and improved functioning; additionally, they can present a $35 \%$ reduction of hospitalization, as well as $37 \%$ decrease of total health costs [29]. In summary, exercise practice can reduce the risk for T2D in adults and elderly, even in the presence of excess of general or central adiposity $[3,11,14,15$, $22,26]$. Therefore, exercise has been considered a primary prevention, low cost and non-pharmacological strategy that can be used in public health initiatives to prevent diabetes in managed care and community setting [1, 22, 29]. Health professionals should encourage individuals of all ages to maintain an active life-style that can attenuate the negative physiologic changes that accompany advancing age, leading to T2D.

The main limitation of this study was that the prevalence of T2D was self-reported. Since diabetes is self-reported, we may be missing cases that are not yet diagnosed. If anything, this would result in an underestimate of the true effect. In addition, considering that this study is cross-sectional it is not possible to provide evidences for causality from our results.

\section{Conclusions}

A direct positive association was found between general and central adiposity, as well as an inverse relation between CRF and exercise practice with T2D. Our findings support that elderly women who practice exercise regularly have lower odds to had T2D. For this reason, health professionals should develop new strategies for primary and secondary prevention for $\mathrm{T} 2 \mathrm{D}$, such as to encourage individuals of all ages to engage on regular exercise practice, which could reduce body fatness and may be beneficial in reducing the prevalence of T2D in older ages.

\section{Conflict of interest}

The authors have declared that no conflict of interest exists.

\section{References}

1. Bassett MT. Diabetes is Epidemic. Am J Publ Health. 2005; 95: 1496.

2. Mokdad AH, Ford ES, Bowman BA, et al. Prevalence of Obesity, Diabetes, and Obesity-Related Health Risk Factors, 2001. JAMA. 2003; 289: 76-79.

3. Sullivan PW, Morrato EH, Ghushchyan V, Wyatt H, Hill JO. Obesity, Inactivity, and the Prevalence of Diabetes and Diabetes-Related Cardiovascular Comorbidities in the U.S., 2000-2002. Diabetes Care. 2005; 28: 1599-1603.

4. Nasri F. Diabetes Mellitus no Idoso. In: Freitas EV et al, eds. Tratado de Geriatria e Gerontologia. Guanabara Koogan, 2002:496-501.

5. Kanaya AM, Harris T, Goodpaster BH, Tylavsky F, Cummings SR. Adipocytokines Attenuate the Association Between Visceral 
Adiposity and Diabetes in Older Adults. Diabetes Care. 2004; 27: 1375-1380.

6. Bloomgarden ZT. Adiposity and Diabetes. Diabetes Care. 2002; 25: 2342-2349.

7. Kahn BB, Flier JS. Obesity and insulin-resistance. J Clin Invest. 2000; 106: 473-481.

8. Boden G. Pathogenesis of type 2 diabetes: insulin resistance. Endocrinol Metab Clin North Am. 2001; 30: 801-815.

9. Rexrode KM, Carey VJ, Hennekens $\mathrm{CH}$, et al. Abdominal adiposity and coronary heart disease in women. JAMA. 1998; 280: 1843-1848.

10. Sharma AM. Adipose tissue: a mediator of cardiovascular risk. Int J Obes. 2002; 26: S5-S7.

11. Weinstein AR, Sesso HD, Lee IM, et al. Relationship of Physical Activity vs Body Mass Index Type 2 Diabetes in Women. JAMA. 2004; 292: 1188-1194.

12. Meisinger C, Löwel H, Thorand B, Döring A. Leisure Time Physical Activity and the risk of type 2 diabetes in men and women from the general population. Diabetologia. 2004; 48: 27-34.

13. Katzmarzyk PT, Church TS, Janssen I, Ross R, Blair SN. Metabolic Syndrome, Obesity, and Mortality - impact of cardiorespiratory fitness. Diabetes Care. 2005; 28: 391-397.

14. Franks PW, Ekelund U, Brage S, Wong M-Y, Wareham NJ. Does the Association of Habitual Physical Activity With the Metabolic Syndrome Differ by Level of Cardiorespiratory Fitness? Diabetes Care. 2004; 27: 1187-1193.

15. Laaksonen DE, Lakka H-M, Salonen JT, Niskanen LK, Rauramaa R, Lakka TA. Low Levels of Leisure-Time Physical Activiy and Cardiorespiratory Fitness Predict Development of the Metabolic Syndrome. Diabetes Care. 2002; 25: 1612-1618.

16. Makrides L, Heigenhauser GJ, Jones NL. High-intensity endurance training in 20- to 30- and 60- to 70-yr-old healthy men. J Appl Physiol. 1990; 69: 2004-2011.

17. Selvin E, Coresh J, Brancati LB. The burden and treatment of diabetes in eldely individuals in the U.S. Diabetes Care. 2006; 29:2415-2419.

18. International Diabetes Federation. Global strategic plan to raise awareness of diabetes. Brussels: IDF; 2003.

19. Ranna JS, Li TY, Manson JE, Hu FB. Adiposity compared with physical inactivity and risk of type 2 diabetes in women. Diabetes Care. 2007; 30:53-58

20. Risvi AA. Management of Diabetes in Older. Am J Med Sci. 2007; 333(1):35-47.

21. Chang AM, Halter JB. Aging and insulin secretion. Am J Physiol Endocrinol Metab. 2003; 284:E7-E12.

22. Church TS, Cheng YJ, Earnest CP, et al. Exercise Capacity and Body Composition as Predictors of Mortality Among Men With Diabetes. Diabetes Care. 2004; 27: 83-88.

23. Rikli RG; Jones CJ. Development and validation of a functional fitness test for community-residing older adults. J Aging Phys Act. 1999; 7: 129-161.

24. Voorrips LE; Ravelli AC; Dongelmans PC; Deurenberg P; Van Staveren WA. A physical activity questionnaire for the elderly. Med Sci Sports Exerc. 1991; 23: 974-979.

25. [Internet] Estatística do Diabetes no Brasil. http://www.diabetes.org.br/imprensa/estatística/numerosno brasil.php

26. Warburton DER, Nicol CW, Bredin SSD. Healthy benefits of physical activity: the evidence. CMAJ. 2006; 174: 801-809.

27. Janssen I, Katzmarzyk PT, Ross R, et al. Fitness Alters the Association of BMI and Waist Circumference with Total and Abdominal Fat. Obesity Research. 2004; 12: 525-537.

28. Wannamethee SG, Shaper AG, Walker M. Overweight and obesity and weight change in middle aged men: cardiovascular disease and diabetes. J Epidemiol Community Health. 2005; 59: 134-139.
29. Nguyen HQ, Ackermann RT, Berke EM, et al. Impact of a Managed-Medicare Physical Activity Benefit on Health Care Utilization and Costs in Older Adults with Diabetes. Diabetes Care. 2007; 30:43-48.

30. Bloomgarden ZT. Consequences of Diabetes. Cardiovascular Disease. Diabetes Care. 2004; 27:1825-1831. 